\title{
Phase-specific Raman spectroscopy for fast segmented microfluidic flows
}

Cite this: Lab Chip, 2014, 14, 2910

\author{
S. K. Luther, S. Will and A. Braeuer*
}

Received 8th April 2014,

Accepted 3rd June 2014

DOI: $10.1039 / c 4 l c 00428 \mathrm{k}$

www.rsc.org/loc

An intensifier based Raman measuring strategy is introduced which allows for a phase-specific signal detection of one single phase in segmented flows at droplet generation frequencies of potentially up to several $\mathrm{kHz}$.

Fluids flowing in transparent microfluidic systems (MFS) can be controlled and handled at well-defined conditions and can be analysed non-invasively using optical measurement techniques. Segmented two-phase microfluidic flows (droplets separated by plugs) can be produced at frequencies up to several kHz. ${ }^{1}$ Therefore, thousands of ultra-small reactors (droplet as confined volume), in which Taylor diffusion is eliminated, sample-surface interactions are reduced and mass transfer and mixing inside the droplets is enhanced, ${ }^{2}$ can be generated and analysed per second in principle. Due to these unique conditions, droplet flows in MFS are attractive for chemical engineering research and applications in general. The fast repetition rate of the flow segmentation (alternating droplets and plugs) requires a measurement technique with high temporal resolution, which then enables the detection of the signals specifically from either the droplets or the plugs.

Unfortunately, the temporal resolution of conventional versions of several optical measurement techniques, such as ultraviolet-visible absorption spectroscopy (UV-vis), Fourier transformed infrared spectroscopy (FTIR), ${ }^{3}$ fluorescence spectroscopy and Raman spectroscopy ${ }^{4}$ is too low. Thus, these have been applied only for the analysis of single-phase fluid flows or slow segmented flows in MFS in material processing- ${ }^{5}$ biological-, pharmaceutical- ${ }^{6,7}$ and reacting systems ${ }^{8,9}$ and for the investigation into mass transfer processes. ${ }^{10-12}$

Here, we present a Raman measurement technique that can probe and accumulate the Raman signals specifically from one phase of the segmented flow (either the droplets or

Lehrstuhl für Technische Thermodynamik (LTT) and Erlangen Graduate School in Advanced Optical Technologies (SAOT), Friedrich-Alexander Universität Erlangen-Nürnberg (FAU), Erlangen, Germany. E-mail: Andreas.Braeuer@fau.de the plugs) and thus is applicable at droplet frequencies of high kHz-rates. Linear Raman techniques suffer from weak scattering cross sections, which are compensated against by integrating the weak Raman signals over a rather long exposure time ${ }^{4}$ on the detector (usually more than one second). This often restricts Raman spectroscopy in multi-phase systems to very slow processes. According to reference, ${ }^{13}$ Raman techniques have only been applied in microfluidic droplet flows if

(1) nanoparticles were added to realize surface enhanced Raman spectroscopy, ${ }^{14}$

(2) the alternating phases are spectrally analysed integrally and it is assumed that the spectra of the two phases do not interfere (immiscible fluids only) and can consequently be separated after the measurement, ${ }^{15,16}$

(3) the flow was stopped after the droplet generation. ${ }^{17}$

In case (1) the presence of nanoparticles may change the chemistry or flow and cases (2) and (3) are applicable for totally immiscible fluids only and render the analysis of mass transfer phenomena between the phases impossible.

The experimental setup of the here proposed phase-specific and fast Raman measuring strategy is sketched in Fig. 1.

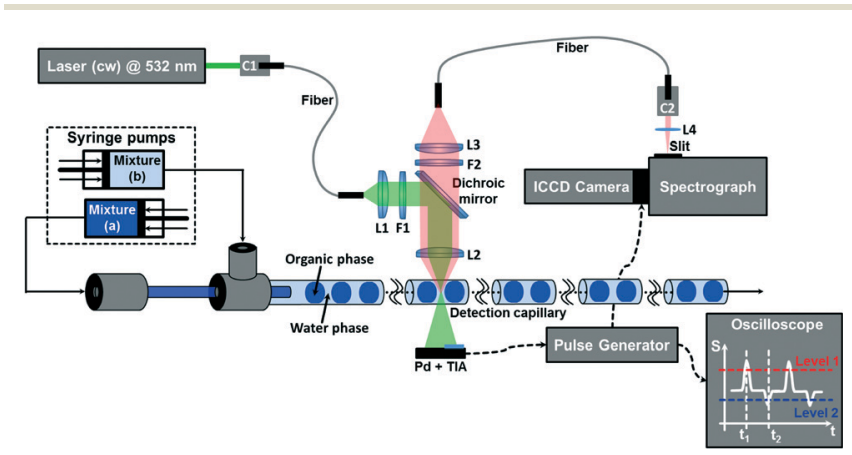

Fig. 1 Scheme of the experimental micro capillary- and optical setup; $C_{1,2}$ : fiber collimators; $L_{1,4}$ : convex lenses; $L_{2,3}$ : achromatic lenses; $F_{1}$ : short pass filter $(550 \mathrm{~nm}) ; F_{2}$ : long pass filter $(535 \mathrm{~nm}) ; \mathrm{Pd}+\mathrm{TIA}$ : photo detector with transimpedance amplifier; $S$ : signal; $t_{1}$ : droplet arriving the focal spot; $t_{2}$ : droplet leaving the focal spot. 
The applied measuring and triggering technique is illustrated and characterized using a model system, where acetone (Ac) is transported between the two almost immiscible compounds, water and ethyl acetate (Ea). Ac was used, as it is completely miscible with water and Ea at room temperature. Mixture (a) which later forms the organic phase (OP) consist of 50/50 vol\% of Ac and Ea, whereas mixture (b) which later forms the water phase (WP) consists of water saturated with Ea. Two syringe pumps (Teledyne ISCO) feed both mixtures to the MFS with total flow rates ranging from 30 to $100 \mu \mathrm{min}^{-1}$, resulting in flow velocities in the detection capillary of 1.3 up to $4.3 \mathrm{~mm} \mathrm{~s}^{-1}$. The "very basic" MFS consists of two concentrically arranged $\mathrm{Si} /$ glass capillaries having an inner diameter of $100 \mu \mathrm{m}$ and $700 \mu \mathrm{m}$, respectively, which induces a co-flow of the two fluids. Downstream of the nozzle, the two fluids mix within few millimetres and afterwards split into two phases to form a stable and periodic flow pattern of alternating OP droplets and WP plugs. In the detection capillary $(L=50 \mathrm{~cm})$, Ac moves from the OP droplets to the WP plugs leading to a reduction and an increase of the Ac concentration in the OP and in the WP, respectively.

A continuous wave Nd:IVO ${ }_{4}$-laser (Millennia, Spectra Physics, $532 \mathrm{~nm}, 250 \mathrm{~mW}$ ) was used for excitation. The laser beam is focused into a fiber, guided to the mobile optical Raman system, subsequently collimated $\left(L_{1}\right)$, cleaned from Si-signal of the fiber $\left(F_{1}=\right.$ short pass filter $\left.550 \mathrm{~nm}\right)$ and focused into the capillary with a focal length of $f_{2}=30 \mathrm{~mm}$. Its mobility enables the probing at various downstream positions inside the detection capillary. The red-shifted Raman signals generated in the focal volume are back scattered and imaged onto a fiber. The dichroic mirror (reflecting wavelengths $<540 \mathrm{~nm}$ and transmitting wavelengths $>540 \mathrm{~nm}$ ) together with the long pass filter $\left(F_{2}\right)$, suppresses the elastically scattered light. The signals are guided with a fiber, focused on the slit (width $=150 \mu \mathrm{m}$ ) of a spectrograph (Andor, Shamrock 303i), spectrally dispersed and detected on the CCD detector of an intensified camera (Andor, iStar DH734-18F-A3). A similar setup without triggering and intensifier is frequently used for macroscopic systems in our lab. ${ }^{18,19}$

The acquisition of Raman spectra specifically from one phase only applied in fast segmented flows was assured by the installation of two measures.

\section{Photo-electric guard}

Aiming at synchronizing the detection of the spectra to the segmented flow, a photo-electric guard (photo detector) is used as it is sketched in Fig. 1, which detects the intensity of the excitation light passing through the capillary. If the phase boundary of a droplet (discontinuous change in refractive index) passes the focal spot of the excitation laser beam, the beam will either be reflected/refracted onto or away from the photo detector. The resulting voltage-signal sequence of the photo-electric guard is visualized on an oscilloscope showing two scenarios (positive or negative peak) for droplets arriving and leaving the focal spot of the laser as sketched in Fig. 1.
This voltage-signal is now transmitted as trigger-in signal to a pulse generator, where two voltage-levels were set to online distinguish between either a WP-plug is replaced by an OP-droplet (level 1) or an OP-droplet is replaced by a WP-plug (level 2).

\section{Photo-electric gate}

The pulse generator triggers the intensifier (photo-electric gate) of the camera to initiate the transmission of the Raman signals to the CCD specifically from either the OP or the WP. Before the droplet (or the plug) is replaced by the following plug (or droplet), the transmission of the Raman signals to the CCD has to be interrupted by the deactivation of the intensifier. The intensifier we used in this study featured minimum gating times of $5 \mathrm{~ns}$ and maximum gating repetition rates of $50 \mathrm{kHz}$.

In summary, the photo-electric guard assigns the pulse generator to activate the intensifier of the intensified CCD camera each time a new droplet (or plug) appears in the laser excitation focus. The intensifier now acts as a photo-electric gate and is kept active only during the pulse duration of the trigger-in pulse and is then deactivated. The pulse duration of the trigger-in pulse must be set short enough to assure that the signals passing the intensifier solely origin from either the droplets or the plugs. The minimum trigger-in pulse duration applied is $5 \mu \mathrm{s}$ and is limited by the resolution of the pulse generator we used. By adjusting the guard-to-gate delay and the pulse width, it is possible to shift the gating time of the intensifier to a specific position inside the droplets or plugs. Consequently, the acquisition of spectra is possible either from the center or from the borders of the droplets/plugs, or from the entire phase. The CCD detector, which is installed behind the intensifier, can now acquire spectra for several seconds and during this time only accumulates the Raman signal intensities of hundreds or thousands of either droplets or plugs.

In Fig. 2, the evolutions of the Ac Raman signal intensity in the OP and the WP for a segmented flow at different

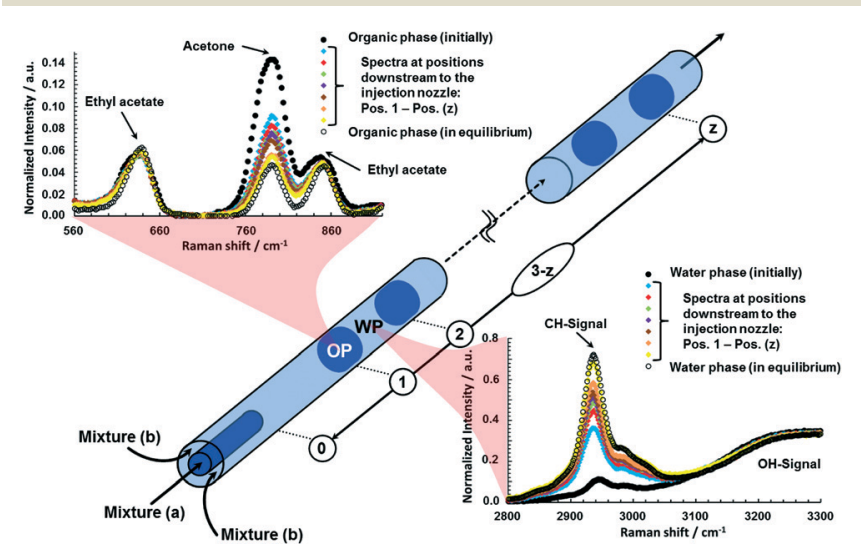

Fig. 2 Decreasing Raman intensity of Ac in the OP - normalized to the Raman intensity of an isolated Ea peak $\left(600-670 \mathrm{~cm}^{-1}\right)$ - and increasing Raman intensity of Ac in the WP - normalized to the Raman intensity of the $\mathrm{OH}$ vibration $\left(3100-3650 \mathrm{~cm}^{-1}\right)$ - for different distances to the nozzle $(0-z)$. 
distances to the nozzle (mobile sensor - immobile MFS) are illustrated exemplarily for flow rates of mixture (a) and (b) of $25 \mu \mathrm{min} \mathrm{min}^{-1}$, which resulted in droplet generation frequencies of approximately $5 \mathrm{~Hz}$.

The spectra in Fig. 2 at one measuring position were acquired consecutively for the OP and the WP with a guardto-gate delay of $40 \mathrm{~ms}$ and a gating time of the intensifier of $40 \mathrm{~ms}$ for a fixed acquisition time of the CCD detector of 3 seconds. During this time the intensifier was set active approximately 15 times ( $5 \mathrm{~Hz}$ droplet generation frequency) for $40 \mathrm{~ms}$, meaning that - during 3 seconds acquisition time of the CCD - the Raman signal was detected over $600 \mathrm{~ms}$ specifically from one phase. (We were not able to produce faster droplet frequencies and therefore demonstrate the applicability of gating frequencies of $40 \mathrm{kHz}$ and gating widths of $5 \mu \mathrm{s}$ further below.) Afterwards, the spectra were normalized to an isolated signal peak of $\mathrm{Ea}$ in case of the $\mathrm{OP}$ and to the $\mathrm{OH}$ vibration of water in case of the WP, reasonably assuming that water and Ea are not traveling across the phase boundary as the water mixture was initially saturated with Ea $\left(7.7 \mathrm{wt} \%{ }^{20}\right)$ and water is sparely soluble in Ea $\left(3.3 \mathrm{wt} \%{ }^{20}\right)$. The spectra of the initial state were acquired by only introducing mixture (a) or (b) to the capillary. The acquisition of the spectra of the equilibrium state was possible by forming a mixture of (a) and (b) inside a cuvette and afterwards introducing only the OP or the WP to the capillary. Finally, the increase of the CH-Raman signal intensity of Ac in the WP, integrated from 2800 to $2973 \mathrm{~cm}^{-1}$ and the decrease of the Raman signal intensity of Ac in the OP, integrated from 700 to $884 \mathrm{~cm}^{-1}$ can be compared and quantified at different positions downstream of the nozzle. With the total volume flow rate and by normalizing the Raman signal intensities to the initial or the equilibrium state, the evolution of the species composition can be given as a function of the residence time for different flow rates, as illustrated in Fig. 3. In Fig. 3, only the evolutions of the Raman signal intensities of Ac are shown as a function of the residence time. For a quantification of the molar composition (not within the scope of this Communication) a calibration would be required, ${ }^{18,21}$ but was not carried out here.

In the following, we experimentally compare the performance of a Raman measurement technique using a

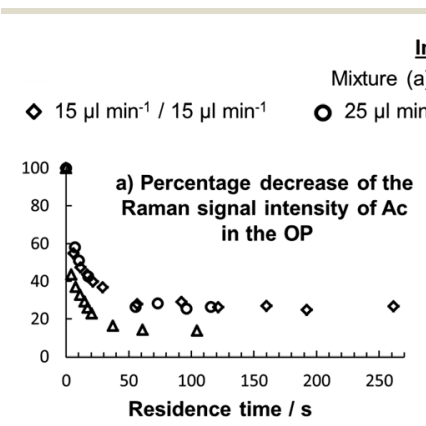

Input

Mixture (a) / Mixture (b)

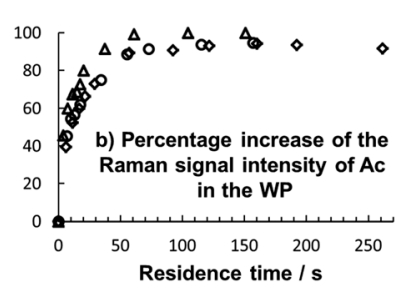

Fig. 3 Evolution of the Raman signal intensity of Ac in the (a) OP and in the (b) WP, as a function of residence time for different flow rates. conventional CCD detector and the one we propose here making use of the intensified/gated CCD detector. As it was not possible to generate a stable periodic segmented flow pattern with droplet generation frequency of $\mathrm{kHz}$ in this basic MFS, the comparison is carried out in a homogeneous singlephase mixture. Now the intensifier is not triggered by the photo-electric guard (no droplets in the flow), but directly by the pulse generator working at a fixed pulse width and frequency.

The shorter the exposure time of the CCD detector (without intensifier), the smaller are the signal-to-noise-ratios (SNR) and the spatial movement of the flow and the higher is the repetition rate of single spectra. 28 droplets or plugs per second can be analyzed at maximum when the exposure time of the CCD detector is set to $18 \mathrm{~ms}$, which is the minimum of the full-frame CCD detector available. This corresponds to a SNR of 28.9 (C-H vibration Raman signal integrated from 2800 to $2973 \mathrm{~cm}^{-1}$ ) and a displacement of the fluid of $78 \mu \mathrm{m}$ for a flow rate of $100 \mu \mathrm{min}^{-1}$ used.

Regarding the intensified CCD, Table 1 shows that an overall signal collection time of $200 \mathrm{~ms}$ can be realized by accumulating four "intensifier active" events $50 \mathrm{~ms}$ each, or forty thousand "intensifier active" events $5 \mu$ s each on the CCD detector. Here, the time between the "intensifier-active" events was set 5 -times the duration of the "intensifier-active" event itself. Therefore, for all experiments summarized in Table 1 the acquisition time of the CCD has been $1 \mathrm{~s}$, while during this time - the intensifier has been activated multiple times for in total $200 \mathrm{~ms}$ and deactivated multiple times for in total $800 \mathrm{~ms}$.

As the effective signal collection time is constant at $200 \mathrm{~ms}$ for all scenarios addressed in Table 1, the SNR does - as expected - not significantly change and is similar to the SNR of the setup using the CCD detector (without intensifier) and the same exposure time (compare Table 1). Nevertheless, multi-phase flows faster by four orders of magnitude can be analyzed in principle with the intensifier strategy (here demonstrated only for single-phase flows), as the movement of the fast flows during the very short gating times of the intensifier is small. Thus, segmented flows with droplet frequencies up to $40000 \mathrm{~Hz}$ can potentially be analyzed phase specifically with the intensifier based Raman detection strategy.

Table 1 Comparison of the SNR and the flow displacement in the capillary at $Q=100 \mu \mathrm{min}^{-1}(\Delta x)$ for different "intensifier active" events triggered $n$-times during the fixed acquisition time of the CCD detector of one second

\begin{tabular}{lrlc}
\hline & & & \\
Intensifier active & \multicolumn{1}{c}{$n$} & & \\
\hline $50 \mathrm{~ms}$ & 4 & 99.0 & $\Delta x / \mu \mathrm{m}$ \\
$1 \mathrm{~ms}$ & 200 & 96.0 & 217 \\
$50 \mu \mathrm{CH}-\mathrm{Cignal}$ & & 4.3 \\
$5 \mu \mathrm{s}$ & 4000 & 95.9 & 0.22 \\
& 40000 & 92.4 & 0.022
\end{tabular}




\section{Conclusions}

In this Communication a Raman technique is suggested and characterized which is generally applicable for all (even nonperiodic) segmented flows in microfluidic systems to which optical access is granted. This technique can be applied to flows, with a droplet generation frequency up to $\mathrm{kHz}$, providing Raman spectra with SNR close to 100 without the addition of nanoparticles to enhance the signal as it is done in SERS. The potential of this screening technique is the extraction of concentration profiles of different phases as a function of the residence time which is one essential quantity to describe a reaction and extraction progress, to quantify the mass transfer or to identify and follow the evolution of a species in biological, chemical or pharmaceutical systems.

\section{Acknowledgements}

The authors gratefully acknowledge funding of the Erlangen Graduate School in Advanced Optical Technologies (SAOT) by the German Research Foundation (DFG) in the framework of the German excellence initiative.

\section{Notes and references}

1 T. Nisisako and T. Torii, Lab Chip, 2008, 8, 287-293.

2 H. Song, D. L. Chen and R. F. Ismagilov, Angew. Chem., Int. Ed., 2006, 45, 7336-7356.

3 K. L. A. Chan, S. Gulati, J. B. Edel, A. J. de Mello and S. G. Kazarian, Lab Chip, 2009, 9, 2909-2913.

4 A. F. Chrimes, K. Khoshmanesh, P. R. Stoddart, A. Mitchell and K. Kalantar-zadeh, Chem. Soc. Rev., 2013, 42, 5880-5906.
5 A. F. Chrimes, A. A. Kayani, K. Khoshmanesh, P. R. Stoddart, P. Mulvaney, A. Mitchell and K. Kalantar-zadeh, Lab Chip, 2011, 11, 921-928.

6 N. Choi, K. Lee, D. W. Lim, E. K. Lee, S.-I. Chang, K. W. Oh and J. Choo, Lab Chip, 2012, 12, 5160-5167.

7 M. Knauer, N. Ivleva, R. Niessner and C. Haisch, Anal. Bioanal. Chem., 2012, 402, 2663-2667.

8 G. Rinke, A. Ewinger, S. Kerschbaum and M. Rinke, Microfluid. Nanofluid., 2011, 10, 145-153.

9 A. Urakawa, F. Trachsel, P. R. von Rohr and A. Baiker, Analyst, 2008, 133, 1352-1354.

10 G. Rinke, A. Wenka, K. Roetmann and H. Wackerbarth, Chem. Eng. J., 2012, 179, 338-348.

11 J.-B. Salmon, A. Ajdari, P. Tabeling, L. Servant, D. Talaga and M. Joanicot, Appl. Phys. Lett., 2005, 86, 094106.

12 M. Wellhausen, G. Rinke and H. Wackerbarth, Microfluid. Nanofluid., 2012, 12, 917-926.

13 Y. Zhu and Q. Fang, Anal. Chim. Acta, 2013, 787, 24-35.

14 M. P. Cecchini, J. Hong, C. Lim, J. Choo, T. Albrecht, A. J. deMello and J. B. Edel, Anal. Chem., 2011, 83, 3076-3081.

15 F. Sarrazin, J.-B. Salmon, D. Talaga and L. Servant, Anal. Chem., 2008, 80, 1689-1695.

16 K. R. Strehle, D. Cialla, P. Rösch, T. Henkel, M. Köhler and J. Popp, Anal. Chem., 2007, 79, 1542-1547.

17 S. E. Barnes, Z. T. Cygan, J. K. Yates, K. L. Beers and E. J. Amis, Analyst, 2006, 131, 1027-1033.

18 S. K. Luther, J. J. Schuster, A. Leipertz and A. Braeuer, J. Supercrit. Fluids, 2013, 84, 146-154.

19 R. Adami, J. Schuster, S. Liparoti, E. Reverchon, A. Leipertz and A. Braeuer, Fluid Phase Equilib., 2013, 360, 265-273.

20 M. Goral, D. G. Shaw, A. Maczynski, B. Goclowska and A. Jezierski, J. Phys. Chem. Ref. Data, 2009, 38, 1093-1127.

21 J. J. Schuster, S. Will, A. Leipertz and A. Braeuer, J. Raman Spectrosc., 2014, 45, 246-252. 